\title{
Inspiration is all around you
}

\author{
Your teachers and your colleagues have an essential role in helping you to determine your scientific \\ interests and your way of doing research, says Giampaolo Pitruzzello.
}

\begin{abstract}
have always been curious about natural phenomena, but I cannot say that I was always passionate about nanoscience. As a teenager, I was very interested in astronomy and this is mainly why I decided to study physics for my degree. After enrolling at the University of Catania, Italy, I was also enrolled at the Scuola Superiore di Catania (SSC), an institute within the University of Catania that offers a high-level education, beyond the standard college programme, an interdisciplinary environment and full scholarships to a limited number of students. There is also the opportunity to choose a tutor who will follow you in your studies and introduce you to scientific research. The tutor I chose, Lucia Romano, is an expert in the synthesis of nanostructures. Following
\end{abstract} her work and her way of doing research I started to develop an interest in phenomena occurring at the nanoscale, and turned away from the mysteries of the universe to investigate those of the very small world.

My first practical introduction to nanoscience occurred during my second year as an undergraduate, when I was involved in a research project for about a year with Lucia Romano and other researchers from the group of Maria Grazia Grimaldi in the Department of Physics and Astronomy. The project concerned the growth of silica nanowires through a vapour-liquid-solid process, which is well known and tested. A thin metal film is deposited onto a silicon substrate, which is then placed in a furnace, in an inert atmosphere. When the temperature of the furnace is raised, the metallic film breaks down into small clusters, which act as catalyst centres for the nanowire formation. During one such experiment we accidentally used a different temperature ramp from the one suggested by the protocol and we observed that the thin metal film, gold in our case, re-assembled as dendritic-shaped nanoparticles, a sort of gold nano-brain reminiscent of fractal structures, while the growth of the nanowires was almost totally suppressed (F. Ruffino, L. Romano, G. Pitruzzello and M. G. Grimaldi, Appl. Phys. Lett. 100, 053102; 2012). These unusual metallic structures could, in principle, be exploited for plasmonic applications. What I've learnt from this experience, apart from the different

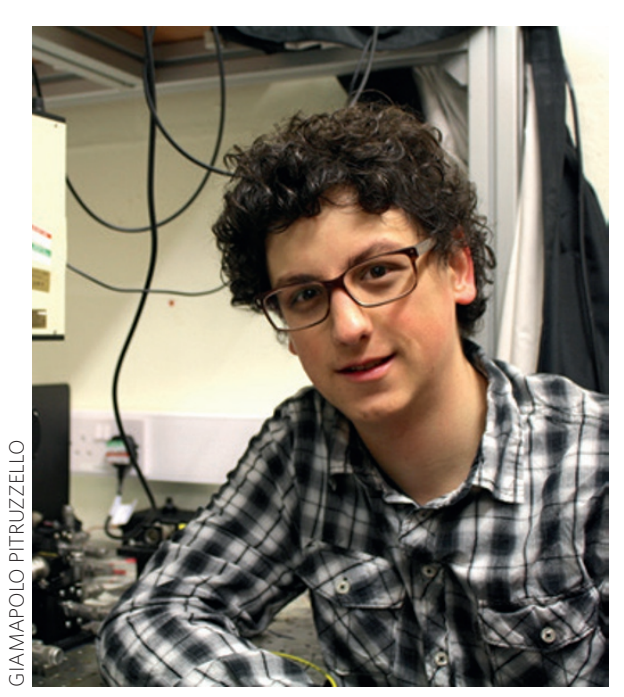

experimental techniques we employed, is that sometimes chance can influence and steer your work in a different direction.

Nanoscience was also central to the rest of my studies. For my master's degree in physics, with a focus on condensed matter, I attended courses on photonics and nanotechnology, semiconductor physics, nanophotonics and nanoplasmonics, and had the fortune of interacting with scientists such as Francesco Priolo, Lucio Claudio Andreani, Matteo Galli and Francesco de Angelis, who were able to convey to students their passion for these subjects. I became really intrigued in how light and matter interact at the nanoscale and especially in the way to control this interaction, mostly because I started to become aware of how the technological development of our society requires continuous miniaturization.

This interest eventually led to my current post at the University of York, in the group of Thomas Krauss, who is a pioneer in the field of nanophotonics, especially in the fabrication of photonic crystals. I am working on the fabrication of silicon bolometers on a silicon-on-insulator platform. A bolometer is a particular type of thermal detector, which, in general, absorbs an optical signal and converts it into an electrical one, by different mechanisms. In a bolometer, the conversion occurs through the dependence of the electrical resistance on temperature. My aim is to realize new structures based on various photonic crystal designs to control the way in which light is absorbed. Some designs and theoretical proposals come from the Hamburg University of Technology, with whom the group collaborates. The fabrication and following characterization processes are long since they require several steps. It involves at least three electron-beam and two ultraviolet lithographies, several etching processes (both wet and dry), one selective doping in a furnace and metal contact evaporation.

This process is beneficial because I am learning all these nanofabrication techniques in detail, and above all, because it makes clear how important it is to split a problem into its simpler parts, whether that be in a clean-room or in an optical lab. I think this is the most effective way to attack and solve a problem because it allows the separation of the variables involved, which can be several dozens of experimental parameters, and to better understand which plays what role and how critical it is in the whole process. When everything has been optimized you can put them back together sequentially and (hopefully) you'll have a working device.

I am also realizing how important, apart from the influence of your teachers, interaction with all members of a scientific team is. I am convinced that brilliant and original solutions can be obtained when people that think in different ways and with a different educational and cultural background work together. This is a lesson I learned at SSC where I lived in an interdisciplinary environment, regularly comparing and discussing with people studying in different fields; my experience in York confirms my belief. The more people I discuss my ideas with, the more perspectives I gain on the same problem, and the more chance I have of solving it efficiently.

GIAMPAOLO PITRUZZELLO is studying
for his master's degree in physics at the
Dipartimento di Fisica e Astronomia,
Università di Catania, via S. Sofia 64, 95123
Catania, Italy, and Scuola Superiore di Catania,
Università di Catania, via Valdisavoia 9, 95123
Catania, Italy. He is currently a visiting student
in the Department of Physics, University of
York, York YO10 5DD, UK.
e-mail: giampaolo.pitruzzello@studium.unict.it 